\title{
Concurrent Ki-67 and p53 Immunolabeling in Cutaneous Melanocytic Neoplasms: An Adjunct for Recognition of the Vertical Growth Phase in Malignant Melanomas?
}

\author{
Zahid Kaleem, M.D., Anne C. Lind, M.D., Peter A. Humphrey, M.D., Ph.D., Robert H. Sueper, M.D., \\ Paul E. Swanson, M.D., Jon H. Ritter, M.D., Mark R. Wick, M.D. \\ Lauren V. Ackerman Laboratory of Surgical Pathology, Washington University Medical Center (ZK, ACL, \\ PAH, PES, JHR), and SmithKline-Beecham Laboratories (RHS), St. Louis, Missouri; and the Robert E. \\ Fechner Laboratory of Surgical Pathology, University of Virginia Health Sciences Center (MRW), \\ Charlottesville, Virginia
}

Ki-67 labeling of paraffin sections has been correlated with the number of cells in non- $G_{o}$ phases of the replicative cell cycle, and this immunohistochemical technique has been applied to the evaluation of a variety of human neoplasms. Similarly, immunolabeling for $\mathrm{p53}$ protein has been used to detect mutations in the corresponding gene, as a reflection of possible cellular transformation in the same context. Both of these techniques were applied to 253 melanocytic tumors of the skin to assess their possible utility in the diagnosis and subcategorization of such lesions. They included 76 banal (common) nevi (CN), 39 Spitz nevi (SN), 62 superficial spreading malignant melanomas in radial growth (SSMMs), 32 nodular malignant melanomas (NMMs), 21 lentigo maligna melanomas in radial growth (LMMs), and 23 melanomas arising in association with preexisting compound nevi (MCN). One hundred cells were counted randomly in each tumor, and dark, exclusively nuclear reactivity was scored as positive labeling; results were recorded as percentages. Negligible Ki-67 and p53 labeling was seen in $\mathrm{CN}$ and $\mathrm{SN}$, at a level that was similar to that obtained in cases of LMM and MCN. The largest proportion of Ki-67-positive and p53-positive cells was observed in NMMs, followed by SSMMs. Radial growth-phase SSMMs and LMMs demonstrated immunoprofiles that were similar to those of melanocytic nevi, and MCN did so as well. The prototypical

Copyright $\odot 2000$ by The United States and Canadian Academy of Pathology, Inc.

VOL. 13, NO. 3, P. 217, 2000 Printed in the U.S.A.

Date of acceptance: July 29, 1999.

Presented in part at the scientific session of the 34th annual meeting of the American Society of Dermatopathology, San Francisco, CA, March 1997.

Address reprint requests to: Mark R. Wick, M.D., Division of Surgical Pathology, Room 3882 Old Medical School, University of Virginia Health Sciences Center, Jefferson Park Avenue, Box 214, Charlottesville, VA 22908; e-mail: mrwick1@usa.net; fax: 804-924-0217. malignant melanocytic tumor representing the vertical growth phase-nodular melanoma-demonstrated a statistically significant difference from all other lesions in this study with respect to Ki-67 index $\left(P=.008, \chi^{2}\right)$ and p53 reactivity $(P<.000001$, $\left.\chi^{2}\right)$. Subsequent concurrent use of a Ki-67 threshold index of $10 \%$ and a p53 index of $5 \%$ correctly indicated the presence of vertical growth in $75 \%$ of NMMs, whereas only $8 \%$ of radial growth phase melanomas of other types were colabeled at the same levels of reactivity for the two markers $(P<$ $\left..00001, \chi^{2}\right)$. Thus, although the distinction between benign and malignant melanocytic tumors could and should not be based on immunohistology for Ki-67 and p53, these results suggest that the latter determinants may, in fact, be used as an adjunct to morphology in the recognition of the vertical growth phase in cutaneous malignant melanomas.

KEY WORDS: Ki-67, Melanocytic neoplasms, Melanoma, Nevi, p53, Skin neoplasms.

Mod Pathol 2000;13(3):217-222

In attempts to extend morphologic observations in the prediction of biologic behavior, several adjunctive techniques have been applied to cutaneous melanocytic lesions of all types. These procedures have included nuclear morphometry (1); immunostaining for p53 proteins (2-4), the INK4A/CDKN2 tumor suppressor gene product (5), proliferating cell nuclear antigen (6-8), or the Ki-67 nuclear protein (9-13); and histochemical analysis of nucleolar organizer regions (14).

A particularly challenging facet of dermatopathology concerns the more narrow setting of melanocytic malignancy. In that context, it is important to identify the growth phases (radial or vertical) of melanomas, in recognition of the prognostic importance attached to them (15). Our laboratory has 
previously studied the expression of putatively mutant p53 protein in melanocytic lesions and found a reasonable correlation between its presence and malignant morphologic and biologic characteristics; moreover, non-wild-type p53 protein was also preferentially associated with vertical growth among melanomas of various types (2). Relatively few reports have addressed the identification of growth phases in cutaneous melanocytic tumors using the MIB1 (anti-Ki-67) antibody (9), and none has discussed its concomitant application with p53 in this context. Moreover, the issue of whether proliferation indices and p53 are truly helpful in the diagnostic distinction between melanocytic nevi and melanomas is still unsettled, in part because of methodological differences in various publications (6-13). Hence, the specific aim of the following evaluation was 2-fold: first, to reassess the utility of concurrent labeling evaluation for Ki-67 and p53 in the differential diagnosis of benign and malignant melanocytic neoplasms, and, second, to study the differences, if any, in the labeling indices for these markers in various biologic subgroups of cutaneous melanoma. The latter included "common" lesions in the radial and vertical growth phases (as defined by Elder \& Murphy [16]), as well as other examples of recognized histologic melanoma variants. A currently advocated microwave-mediated enhancement method (17) was used to optimize exposure of the Ki-67 and p53 target epitopes in standard paraffin sections.

\section{MATERIALS AND METHODS}

A total of 253 well-characterized cutaneous melanocytic neoplasms were studied, as obtained from the surgical pathology files of Washington University Medical Center and SmithKline-Beecham Laboratories (St. Louis, Missouri). These specimens were procured as excisional biopsies from 101 males and 153 females, ranging in age from 3 to 98 years, whose lesions had arisen in various locations. The samples were obtained with standard surgical technique and immediately fixed in neutralbuffered formalin. Each of these tumors had been diagnostically classified originally by at least two of the authors using established morphologic criteria (16). Vertical growth in melanomas was defined by the presence of at least one of the following criteria (16): all tumor cell nests in the epidermis being smaller than at least one in the dermis, differing cytologic appearances of the intraepidermal and intradermal components, the presence of dermal tumor cell nests of 25 or more cells in width, and mitotic activity in intradermal neoplastic cells. Complete follow-up information was obtained in all instances to validate the nosologic process.
The various tumors were categorically distributed as follows: 76 common nevi (CN), 39 Spitz nevi (SN), 62 superficial spreading malignant melanomas (SSMMs), 32 nodular malignant melanoma (NMMs), 21 lentigo maligna melanomas (LMMs), and 23 melanomas arising in association with preexisting common nevi (MCN). Each of the nodular melanomas was, by definition, in the vertical growth phase, whereas all other melanomas in the study were, by design, radial growth phase lesions.

Paraffin sections of all specimens were cut at $4 \mu$ thickness and mounted on glass slides coated with poly-L-lysine (Sigma-Aldrich Chemical Co., St. Louis, MO). They were dewaxed in xylene and absolute ethanol; incubated for $30 \mathrm{~min}$ in methanolic $\mathrm{H}_{2} \mathrm{O}_{2}(0.3 \%[\mathrm{v} / \mathrm{v}])$ to quench endogenous peroxidase; and rehydrated by passage through graded ethanol solutions, distilled water, and phosphatebuffered-saline (PBS; pH 7.4). Heat-mediated "antigen retrieval” (17) was undertaken after immersion of the slides in standard citrate buffer $(0.01 \mathrm{M}$; $\mathrm{pH}$ 6.0). They were warmed for $12 \mathrm{~min}$ in a microwave oven (Sharp, Los Angeles, CA; $600 \mathrm{~W} ; 2,450$ $\mathrm{MHz}$ ). After a 20-min cooling period, the sections were rinsed in water and incubated in PBS. The MIB-1 antibody to Ki-67 (Dako Co., Carpinteria, CA; dilution 1:300) and a "cocktail" of anti-p53 reagents (clone designations 1801 and D07; BioGenex Co., Dublin, CA, and Dako, respectively) were then applied and the sections were incubated for 16 hours at $4^{\circ} \mathrm{C}$. Antibody bridge assembly with the avidinbiotin-peroxidase complex (ABC) technique, using the Elite ABC kit (Vector Laboratories; Burlingame, CA), was carried out on the next day by sequential 1 -h incubations. Chromogenic development was obtained by immersion of the sections in nickel chloride-complexed 3,3'-diaminobenzidine solution $\left(0.25 \mathrm{mg} / \mathrm{mL}\right.$ with $\left.0.003 \% \mathrm{H}_{2} \mathrm{O}_{2}\right)$ for a maximum of $10 \mathrm{~min}$. The slides were then lightly counterstained with Harris's hematoxylin.

Positive controls for Ki-67 studies were represented by paraffin sections of "stock" neoplasms that had been previously studied by flow cytometry to determine proportions of tumor cells in the different phases of the cell cycle. Similar sections of neoplasms that had been shown to manifest p53 mutations by Southern blot analysis also were used as positive controls. Nonimmune murine ascitic fluid was substituted for the primary antibodies as a negative control. Cells were considered Ki-67 positive or p53 positive when they demonstrated strong and exclusively nuclear labeling. Those cases with no reactivity whatsoever were regarded as negative, whereas immunoreactivity in the others was scored semiquantitatively (as " 1 to $4 \%+$," " 5 to $9 \%+$," and $\geq 10 \%+$, etc.) by assessment of 100 tumor cells in randomly selected microscopic fields at $400 \times$ magnification. Paired categorical results were com- 
pared by $\chi^{2}$ analysis, using True Epistat statistical computer software (Epistat Services, Richardson, TX). Similarly, a Bayesian formula was used to calculate the predictive value of "positive" results, as compared with correlation with morphologic features of the vertical growth phase.

\section{RESULTS}

None of the common nevi showed more than 5\% labeling for Ki-67, and all of them were completely nonreactive for p53 as well. The mean MIB-1 index was $0.5 \%$ in that group, with a range of 0 to $5 \%$ reactive cells (Tables 1 and 2). The majority of cases of CN (43 of 76 [57\%]) failed to exhibit any nuclear staining. Likewise, SN demonstrated a very low Ki-67 index, ranging from 0 to $11 \%$ with a mean of $1.4 \%$ (Fig. 1); only one case of SN manifested more than 5\% nuclear labeling. All SN likewise failed to label for p53. The results for LMMs and MCN were similar to those obtained in common nevi and $\mathrm{SN}$; the average MIB-1 indices for those two forms of melanoma were $1.5 \%$ and $0.6 \%$, respectively, and only $5 \%$ of LMMs and $4 \%$ of MCN were labeled for p53. The highest degree of both Ki-67 and p53 immunoreactivity was observed in NMMs. Seventyeight percent of those tumors were p53 positive, and they had a mean proliferation index of $16.1 \%$ (Figs. 2 and 3). The only other group of melanocytic tumors with substantial p53 labeling $(25 \%)$ was the SSMMs, in which an average Ki-67 staining index of $3.1 \%$ was obtained (Fig. 4). In aggregate, the proliferation indices of nodular melanoma and superficial spreading melanomas differed statistically from those of LMMs and melanomas arising in preexisting nevus $\left(P<.05, \chi^{2}\right)$, but this obviously was due principally to the effect of data from the NMM group. Further corroboration of the latter point was gained from the observation that a statistically significant difference was also observed in the mean Ki-67 indices between SSM and NMM $\left(P<.05, \chi^{2}\right)$. Conversely, p53 labeling was significantly and independently different for both the SSMM and NMM categories as compared with all other melanocytic lesions in the study $\left(P=.035, \chi^{2}\right)$ (Fig. 5). Using a model with a $10 \%$ cutoff level for MIB-1 labeling and a 5\% level for concurrent p53 immunoreactivity, $24(75 \%)$ of the NMMs in this series could be correctly identified as in the vertical growth phase. In contrast, only five (8\%) of the radial growth phase SSMMs showed similar findings in regard to the two markers being considered (Fig. 6). In the context of all melanomas in this study, the positive predictive value of the previously cited p53/Ki-67 threshold was found to be $83 \%$ for identification of the vertical growth phase.

\section{DISCUSSION}

Various monoclonal antibodies against the Ki-67 nuclear antigen have been used to measure the proliferative index (PI) in malignant melanomas, and correlations have been drawn between that analyte and tumor thickness, metastatic potential, or overall prognosis (18-20). Fewer studies have applied PI as a discriminative tool in separating melanocytic nevi from malignant melanomas, and, with some exceptions, most of those analyses were based on a limited number of cases or they used dissimilar methods, some of which would be impractical in routine diagnostic pathology $(6,21-25)$. Our goals in part reproduced those of earlier assessments of PI in melanocytic lesions, but they were also somewhat different. We not only considered the value of Ki-67 labeling in the cited differential diagnosis but also evaluated its potential role in recognition of the vertical growth phase in undisputed melanomas and paired it with concomitant p53 immunolabeling toward the same end. Growth phase in melanocytic malignancies has great significance in the estimation of overall prognosis, as recently summarized (26).

Using Ki-S5, another immunohistologic marker of PI, Rudolph et al. (12) suggested that a growth fraction of $5 \%$ could be used to help discriminate between benign and malignant melanocytic proliferations. Our results (and conclusions) deviate to some extent from those of the latter study, probably because a different indicator of proliferative capacity was used; that reality underscores that each monoclonal antibody used in PI studies (e.g., antiproliferating cell nuclear antigen, MIB-1, Ki-S5)

TABLE 1. Ki-67 Labeling in 253 Melanocytic Lesions of the Skin

\begin{tabular}{lcccccc}
\hline & $\begin{array}{c}\text { CN } \\
(n=76)\end{array}$ & $\begin{array}{c}\text { SN } \\
(n=39)\end{array}$ & $\begin{array}{c}\text { SSMM } \\
(n=62)\end{array}$ & $\begin{array}{c}\text { NMM } \\
(n=32)\end{array}$ & $\begin{array}{c}\text { LMM } \\
(n=21)\end{array}$ & $\begin{array}{c}\text { MCN } \\
(n=23)\end{array}$ \\
\hline Range of labeling (\% cases) & $0-5$ & $0-11$ & $0-21$ & $5-56$ & $0-7$ & $0-5$ \\
Mean labeling (\% cases) & 0.5 & 1.4 & 3.1 & 16.1 & 1.5 & 0.6 \\
Absence of labeling (\# cases) & 43 & 20 & 7 & 0 & 11 & 9 \\
1-5\% positivity (\# cases) & 33 & 18 & 42 & 3 & 1 & 0 \\
6-9\% positivity (\# cases) & 0 & 0 & 9 & 25 & 0 \\
$\geq 10 \%$ positivity (\# cases) & 0 & 1 & 4 & 0 \\
\hline
\end{tabular}

CN, common nevi; SN, Spitz nevi; SSMM, superificial spreading malignant melanoma; NMM, nodular malignant melanoma; LMM, lentigo maligna melanoma; MCN, melanoma arising in compound nevi. 


\begin{tabular}{|c|c|c|c|c|c|c|}
\hline & $\begin{array}{c}\mathrm{CN} \\
(n=76)\end{array}$ & $\begin{array}{c}\text { SN } \\
(n=39)\end{array}$ & $\begin{array}{c}\text { SSMM } \\
(n=62)\end{array}$ & $\begin{array}{c}\text { NMM } \\
(n=32)\end{array}$ & $\begin{array}{c}\text { LMM } \\
(n=21)\end{array}$ & $\begin{array}{c}\text { MCN } \\
(n=23)\end{array}$ \\
\hline Range of labeling (\% cases) & 0 & 0 & $0-18$ & $0-49$ & $0-6$ & $0-4$ \\
\hline Mean labeling (\% cases) & 0 & 0 & 2.1 & 12.6 & 0.8 & 0.3 \\
\hline Absence of labeling (\# cases) & 76 & 39 & 15 & 1 & 15 & 19 \\
\hline 1-4\% positivity (\# cases) & 0 & 0 & 40 & 7 & 7 & 4 \\
\hline 5-9\% positivity (\# cases) & 0 & 0 & 9 & 6 & 1 & 0 \\
\hline$\geq 10 \%$ positivity (\# cases) & 0 & 0 & 1 & 18 & 0 & 0 \\
\hline
\end{tabular}

CN, common nevi; SN, Spitz nevi; SSMM, superificial spreading malignant melanoma; NMM, nodular malignant melanoma; LMM, lentigo maligna melanoma; MCN, melanoma arising in compound nevi.

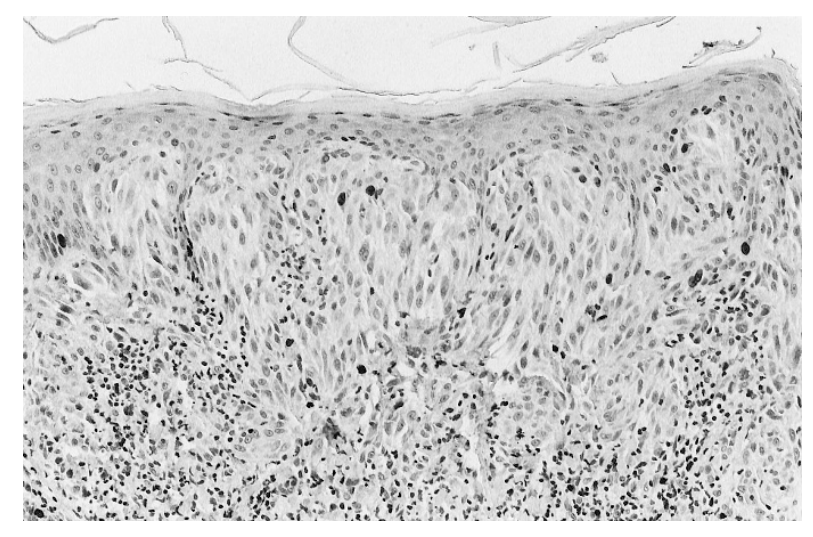

FIGURE 1. Limited Ki-67 labeling in Spitz nevus, showing immunoreactivity in fewer than $3 \%$ of the tumor cells (hematoxylin \& eosin, $160 \times)$.

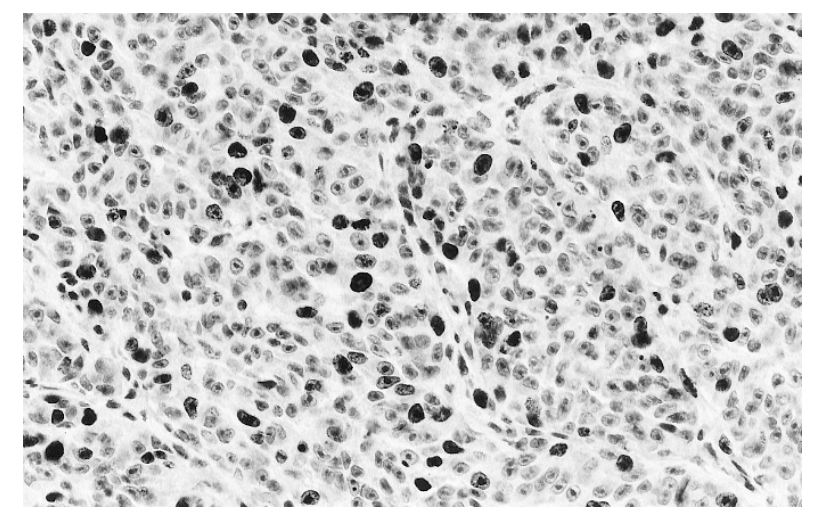

FIGURE 2. Nodular melanoma, showing p53 immunoreactivity in approximately $7 \%$ of tumor cells (anti-p53 immunostain, $300 \times$ ).

shows its own distinctive "profile" in paraffin sections of various neoplastic tissues, and those reagents therefore cannot be compared with one another directly $(27,28)$. In the Rudolph et al. (12) study, a mean PI of $6.38 \%$ was seen in 57 in situ melanomas, and that was significantly different from the Ki-S5 index in benign nevi. In contrast, we found no statistically meaningful differences in MIB-1 labeling between a spectrum of melanocytic nevi ( $\mathrm{CN}$ and $\mathrm{SN})$ and de novo LMMs or melanomas arising in $\mathrm{CN}$. Those results may be considered surprising by some observers, considering the different biologic natures of the lesions in question. However, it should be realized that although label-

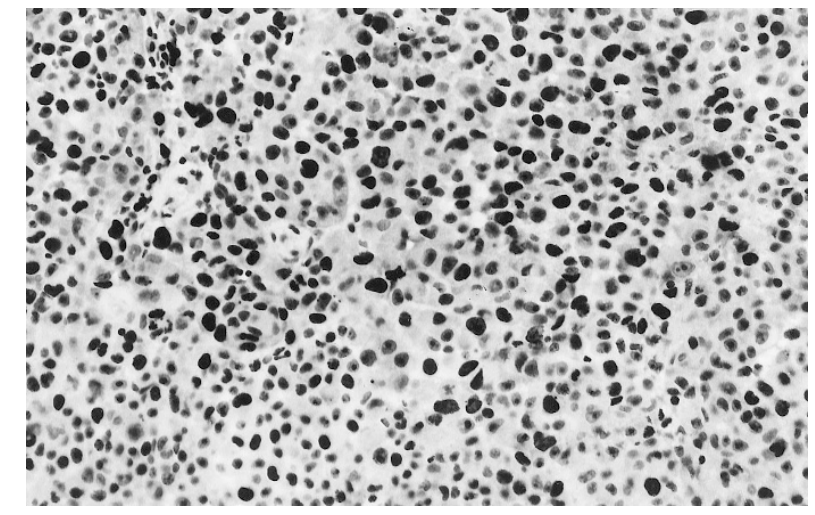

FIGURE 3. Approximately $50 \%$ of the neoplastic cells in this nodular melanoma are labeled by Ki-67 (Ki-67 immunostain, 300×).

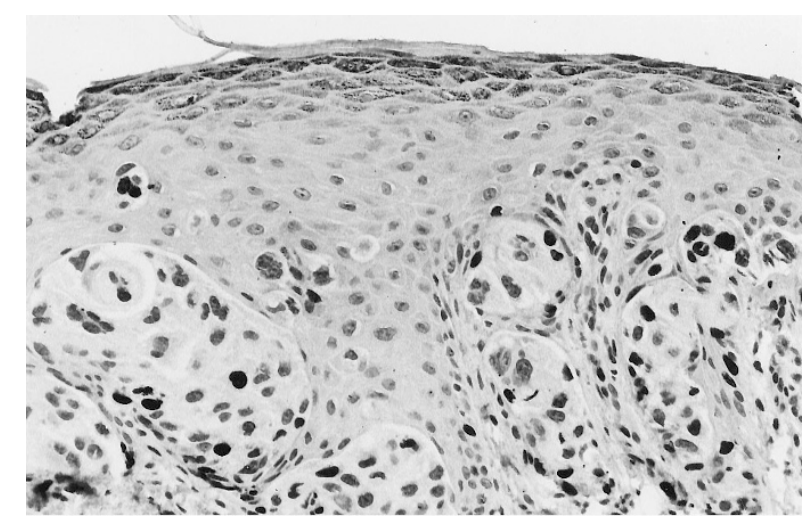

FIGURE 4. Fewer than $3 \%$ of the neoplastic cells in the dermis are labeled by Ki-67 in this superficial spreading melanoma. Virtually identical results were obtained in the same case with anti-p53 immunostains (Ki-67 immunostain, 200×).

ing with MIB-1 correlates well with the number of cells in non- $\mathrm{G}_{0}$ phases of the cell cycle, that quantum is probably not very different in benign nevi and radial growth phase melanomas. Indeed, low PIs in the latter group of neoplasms are consonant with their clinical attributes, which include a relatively long chronological evolution and a concurrently prolonged incapability for metastasis (29). The predominant lack of putatively mutant p53 protein in those lesions is also consistent with such attributes, inasmuch as the p53 gene in melanocytic lesions is thought to be aberrant mainly in malignant tumors that have acquired metastatic 


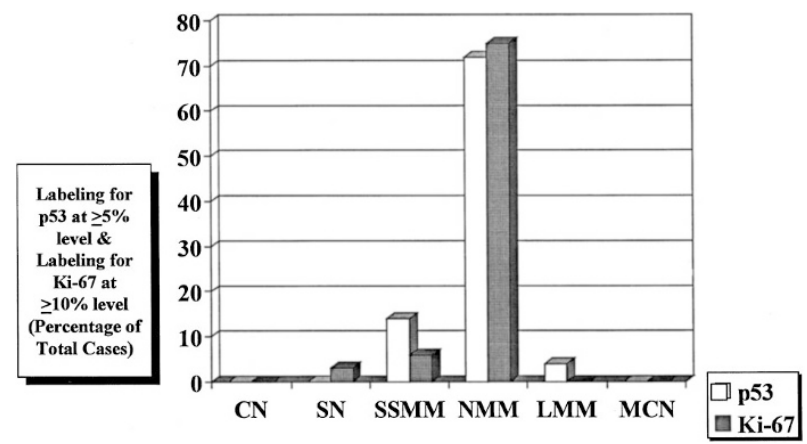

FIGURE 5. Proportions of melanocytic lesions in this study labeling for p53 and Ki-67 at $\geq 5 \%$ and $\geq 10 \%$ levels, respectively. CN, common nevi; SN, Spitz nevi; SSMM, superficial spreading malignant melanoma; NMM, nodular malignant melanoma; LMM, lentigo maligna melanoma; MCN, melanoma in preexisting compound nevi).

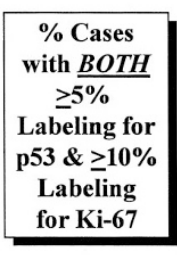

FIGURE 6. Percentages of cases in this study that were labeled concurrently for p53 and Ki-67 at $\geq 5 \%$ and $\geq 10 \%$ levels. CN, common nevi; SN, Spitz nevi; SSSM, superficial spreading malignant melanoma; NMM, nodular malignant melanoma; LMM, lentigo maligna melanoma; MCN, melanoma in preexisting compound nevi).

potential (i.e., entered the vertical growth phase) (2-4).

Melanomas arising in MCN have received comparatively little attention in the pertinent literature (30); they are characterized by the appearance of a subpopulation of obviously malignant melanocytes, arising in the background of a "parent" nevus. The malignant component in MCN may be junctional or intradermal. The PIs and p53 profiles of the two distinct elements of such tumors have been heretofore unstudied, and they again seem to be similar to one another. In the broader context of systemic neoplasia, however, it is known that PI and gene mutations are related to the number of intragenomic "lesions" that have accrued, with consequent dysregulation of cellular replication $(31,32)$. At least at an immunohistochemical level, there is evidence for very infrequent mutation in the $p 53$ tumor suppressor gene in both radial growth LMMs and MCN (2). Therefore, the coincidence of PIs in those two tumor groups is not altogether unexpected, and, although unsubstantiated by rigorous clinical analyses, that observation further suggests that most MCN are closely allied biologically to radial growth phase melanomas of more "conventional" morphotypes.

The mean Ki-67 labeling index observed in radial growth phase SSMMs was higher than that of comparable LMMs and MCN, but it was still well below the PI of vertical growth phase (nodular) melanomas in this series. Such data contrast with the findings of Fogt and co-workers (14), who reported no statistically significant differences in MIB-1 labeling of 12 radial growth phase melanomas and 8 vertical growth phase tumors; however, that study was clearly limited by its small number of cases. In fact, we believe that our results could be anticipated in light of accepted knowledge concerning the biology of growth phases in melanomas, as well as the observed p53 labeling data obtained in this series (15). Vertical growth (exemplified by NMMs) is accompanied by alterations in DNA content, amplification or suppression of a variety of gene complexes, and synthesis of various proteins that facilitate stromal invasion and distant metastasis $(15,26)$. Hence, it would be logical to expect that proliferation in that growth phase would diverge from that seen in radial growth melanomas.

On the basis of our observations, we believe that the most justifiable diagnostic use of p53 and MIB-1 indices in melanocytic lesions rests not on their association with benignancy or malignancy (33) but rather on their application as adjuncts (preferably in combination with one another) in the objective diagnostic distinction between radial and vertical growth phases of melanoma. Because exceptions do occur and some minor overlap was indeed observed in the immunophenotypes of SSMM and NMM, some caution should be exercised in regard to such evaluations. P53/MIB-1 immunostaining results should be integrated with available morphologic and clinical information and used only as an interpretative supplement. The construction of this preliminary evaluation did not encompass melanomas with an "indeterminate" growth phase, as reflected by their morphologic characteristics. Indeed, a validation study to test the prospective utility of our results has been initiated, with particular emphasis on cases in the latter category. Obviously, clinical outcomes analysis of a large group of lesions will be necessary to corroborate the value of Ki-67 and p53 immunoreactivity in a determination of the growth phases of melanomas.

\section{REFERENCES}

1. Talve LA, Collan YU, Ekfors TO. Nuclear morphometry, immunohistochemical staining with Ki-67 antibody, and mitotic index in the assessment of proliferative activity and prognosis of primary malignant melanomas of the skin. J Cutan Pathol 1996;23:335-43.

2. Kaleem Z, Lind AC, Humphrey PA, Swanson PE, Sueper R, Ritter JH, et al. Immunohistochemical evaluation of puta- 
tively mutant p53 protein in cutaneous melanocytic neoplasms: a study of 256 cases. Int J Surg Pathol 1998;6:73-80.

3. Sparrow LE, Soong R, Dawkins HJ, Iocopetta BJ, Heenan PJ. P53 gene mutation and expression in nevi and melanomas. Melanoma Res 1995;5:93-100.

4. Cristofolini M, Boi S, Girlando S, Zumiani G, Cristofolini P, Dalla Palma P, et al. P53 protein expression in nevi and melanomas. Arch Dermatol 1993;129:739-43.

5. Talve LA, Sauroja I, Collan YU, Punnonen K, Ekfors T. Loss of expression of the p16INK4/CDKN2 gene in cutaneous malignant melanoma correlates with tumor cell proliferation and invasive stage. Int J Cancer 1997;74:255-9.

6. Rieger E, Hofmann-Wellenhof R, Soyer HP, Kofler R, Cerroni L, Smolle J, et al. Comparison of proliferative activity as assessed by proliferating cell nuclear antigen (PCNA) and Ki-67 monoclonal antibodies in melanocytic skin lesions: a quantitative immunohistochemical study. J Cutan Pathol 1993;20:229-36.

7. Tu P, Miyauchi S, Miki Y. Proliferative activities in Spitz nevus compared with melanocytic nevus and malignant melanoma using expression of PCNA/cyclin and mitotic rate. Am J Dermatopathol 1993;15:311-4.

8. Bjornhagen V, Bonfoco E, Brahme EM, Lindholm J, Auer G. Morphometric, DNA, and proliferating cell nuclear antigen measurements in benign melanocytic lesions and cutaneous malignant melanoma. Am J Dermatopathol 1994;16:615-23.

9. Korabiowska M, Brinck U, Mirecka J, Kellner S, Marx D, Schauer A. Antigen Ki-67 and c-myc oncogene as related to histochemical parameters in pigmented skin lesions. In Vivo 1995;9:433-8.

10. Soyer HP. Ki-67 immunostaining in melanocytic skin tumors: correlation with histologic parameters. J Cutan Pathol 1991;18:264-72.

11. Smolle J, Soyer HP, Kerl H. Proliferative activity of cutaneous melanocytic tumors defined by Ki-67 monoclonal antibody: a quantitative immunohistochemical study. Am J Dermatopathol 1989;11:301-7.

12. Rudolph P, Schubert C, Schubert B, Parwaresch R. Proliferation marker Ki-S5 as a diagnostic tool in melanocytic lesions. J Am Acad Dermatol 1997;37:169-78.

13. Wayers W, Bonczkowitz M, Wayers I, Bittinger A, Schill WB. Melanoma in situ versus melanocytic hyperplasia in sundamaged skin: assessment of the significance of histopathologic criteria for differential diagnosis. Am J Dermatopathol 1996;18:560-6.

14. Fogt F, Vortmeyer AO, Tahan SR. Nucleolar organizer regions (AgNOR) and Ki-67 immunoreactivity in cutaneous melanocytic lesions. Am J Dermatopathol 1995;17:12-7.

15. Imber MJ, Mihm MC Jr. Biological, prognostic significance of vertical growth phase characteristics in malignant melanoma. Monogr Pathol 1988;30:19-34.

16. Elder DE, Murphy GF. Melanocytic tumors of the skin. In: Rosai J, editor. Atlas of tumor pathology, Series 3, Fascicle 2. Bethesda, MD: Armed Forces Institute of Pathology/Universities Associated for Research \& Education in Pathology; 1991. pp. 103-205.

17. Cattoretti G, Pileri S, Parravicini C, Becker MH, Poggi S,
Bifulco C, et al. Antigen unmasking on formalin-fixed, paraffin-embedded tissue sections. J Pathol 1993;171:83-98.

18. Vogt T, Zipperer KH, Vogt A, Holzel D, Landthaler M, Stolz W. P53 protein and Ki-67 antigen expression are both reliable biomarkers of prognosis in thick stage I nodular melanomas of the skin. Histopathology 1997;30:57-63.

19. Boni R, Doguoglu A, Burg G, Muller B, Dummer R. MIB-1 immunoreactivity correlates with metastatic dissemination in primary thick cutaneous melanoma. J Am Acad Dermatol 1996;35:416-8.

20. Ramsay JA, From L, Iscoe NA, Kahn HJ. MIB-1 proliferative activity is a significant prognostic factor in primary thick cutaneous melanomas. J Invest Dermatol 1995;105:22-6.

21. McNutt NS, Urmacher C, Hakimian J, Hoss DM, Lugo J. Nevoid malignant melanoma: morphologic patterns and immunohistochemical reactivity. J Cutan Pathol 1995;22:50217.

22. Mehregan DR, Mehregan DA, Mehregan AH. Proliferating cell nuclear antigen staining in deep penetrating nevi. J Am Acad Dermatol 1995;33:685-7.

23. Penneys NS, Seigfried E, Nahass G, Vogler C. Expression of proliferating cell nuclear antigen in Spitz nevus. J Am Acad Dermatol 1995;32:964-7.

24. Tokuda Y, Mukai K, Matsuno Y, Furuya S, Shimosato Y, Takasaki Y, et al. Proliferative activity of cutaneous melanocytic neoplasms defined by a proliferating cell nuclear antigen labeling index. Arch Dermatol Res 1992;284:319-23.

25. Niemann TH, Argenyi ZB. Immunohistochemical study of Spitz nevi and malignant melanoma with use of antibody to proliferating cell nuclear antigen. Am J Dermatopathol 1993; 15:441-5.

26. Wick MR. Prognostic factors for cutaneous melanoma. Am J Clin Pathol 1998;110:713-8.

27. Coltrera MD, Skelly M, Gown AM. Anti-PCNA antibody PC10 yields unreliable proliferation indexes in routinely processed, deparaffinized, formalin-fixed tissue. Appl Immunohistochem 1993;1:193-200.

28. Mangiarotti R, Bottone MG, Danova M, Pellicciari C. Bivariate flow cytometric analysis of DNA content versus immunopositivity for ribonucleotide reductase M1 subunit in the cell cycle. Cytometry 1998;32:78-85.

29. Elder DE, Guerry D IV, Epstein MN, Zehngebot L, Lusk E, Van Horn M, et al. Invasive malignant melanomas lacking competence for metastasis. Am J Dermatopathol 1984; 6(Suppl):55-61.

30. Tajima Y, Nakajima T, Sugano I, Nagao K, Kondo Y. Malignant melanoma within an intradermal nevus. Am J Dermatopathol 1994;16:301-6.

31. Kamb A, Herlyn M. Malignant melanoma. In: Vogelstein B, Kinzler KW, editors. The genetic basis of human cancer. New York: McGraw-Hill; 1998. pp. 507-18.

32. Asschert JG, Vellenga E, DeJong S, DeVries EG. Mutual interactions between p53 and growth factors in cancer. Anticancer Res 1998;18:1713-25.

33. Kanter-Lewensohn L, Hedblad MA, Wejde J, Larsson O. Immunohistochemical markers for distinguishing Spitz nevi from malignant melanomas. Mod Pathol 1997;10:917-20. 